\title{
Influence of Macrocolumnar EEG on Ca Waves
}

\author{
Lester Ingber
}

\begin{abstract}
A "smoking gun" for explicit top-down neocortical mechanisms that directly drive bottom-up processes that describe memory, attention, etc. The top-down mechanism considered are macrocolumnar EEG firings in neocortex, as described by a statistical mechanics of neocortical interactions (SMNI), developed as a magnetic vector potential $A$. The bottom-up process considered are $\mathrm{Ca}^{2+}$ waves prominent in synaptic and extracellar processes that are considered to greatly influence neuronal firings. Here, the complimentary effects are considered, i.e., the influence of $\mathrm{A}$ on $\mathrm{Ca}^{2+}$ momentum, p. The canonical momentum of a charged particle in an electromagnetic field, $\boldsymbol{\Pi}=\mathbf{p}+q \mathbf{A}$ (SI units), is calculated, where the charge of $\mathrm{Ca}^{2+}$ is $q=2 e, e$ is the magnitude of the charge of an electron, valid in both classical and quantum mechanics. It is shown that $A$ is large enough to influence $p$. This suggests that, instead of the common assumption that $\mathrm{Ca}^{2+}$ waves contribute to neuronal activity, they may in fact at times be caused by the influence of A of larger-scale EEG.
\end{abstract}

Index Terms-short-term memory, astrocytes, neocortical dynamics, vector potential

\section{SMOKIng Gun FOR TOP-Down Processes}

There is a growing awareness of the importance of multiple scales in many physical and biological systems, including neuroscience [1], [2]. As yet, there does not seem to be any "smoking gun" for explicit top-down mechanisms that directly drive bottom-up processes that describe memory, attention, etc. Of course, there are many top-down type studies demonstrating that neuromodulator [3] and neuronal firing states, e.g., as defined by EEG frequencies, can modify the milieu or context of individual synaptic and neuronal activity, which is still consistent with ultimate bottom-up paradigms. However, there is a logical difference between top-down milieu as conditioned by some prior external or internal conditions, and some direct top-down processes that direct cause bottom-up interactions specific to STM. Here, the operative word is "cause".

\section{A. Magnetism Influences in Living Systems}

There is a body of evidence that suggests a specific topdown mechanism for neocortical STM processing.

An example of a direct physical mechanism that affects neuronal processing not part of "standard" sensory influences is the strong possibility of magnetic influences in birds at quantum levels of interaction [4]-[6]. It should be noted that this is just a proposed mechanism [7].

The strengths of magnetic fields in neocortex may be at a threshold to directly influence synaptic interactions with astrocytes, as proposed for long-term memory (LTM) [8] and short-term memory (STM) [9], [10] Magnetic strengths associated by collective EEG activity at a columnar level gives

Lester Ingber is President of Lester Ingber Research in Ashland, Oregon USA. (e-mail: ingber@alumni.caltech.edu) rise to even stronger magnetic fields. Columnar excitatory and inhibitory processes largely take place in different neocortical laminae, providing possibilities for more specific mechanisms.

\section{SMNI CONTEXT}

Since 1981 about 30 papers on a statistical mechanics of neocortical interactions (SMNI) has been detailed properties of short-term memory, long-term memory, EEG analyses, and other properties of neocortex [11]-[16].

This discussion compares the momentum of a $\mathrm{Ca}^{2+}$ ion with macrocolumnar EEG fields. Columnar EEG firings calculated by SMNI lead to electromagnetic fields which can be described by a vector potential 4-vector [17]. In the standard gauge, the 3-vector components of this vector potential describe magnetic fields, denoted here as $\mathbf{A}$, are of interest. In this context this is referred to as the SMNI vector potential (SMNI-VP). An early discussion of SMNI-VP contained in a review of short-term memory as calculated by SMNI was not as detailed [16]. Note that gauge of $\mathbf{A}$ is not specified here, and this can lead to important effects especially at quantum scales [18]. Current research is directed to more detailed interactions of SMNI-VP firing states with $\mathrm{Ca}^{2+}$ waves.

This paper concerns a dipole model for collective minicolumnar oscillatory currents, corresponding to top-down signaling, flowing in ensembles of axons, not for individual neurons. The top-down signal is claimed to cause relevant effects on the surrounding milieu, but is not appropriate outside these surfaces due to strong attenuation of electrical activity. However, the vector potentials produced by these dipoles due to axonal discharges do survive far from the axons, and this can lead to important effects at the molecular scale, e.g., in the environment of ions [19], [20].

The SMNI columnar probability distributions, derived from statistical aggregation of synaptic and neuronal interactions among minicolumns and macrocolumns, have established credibility at columnar scales by detailed calculations of properties of STM. Under conditions enhancing multiple attractors, detailed in SMNI papers with a "centering mechanism" effected by changes in background synaptic activity, multiple columnar collective firing states are developed. It must be stressed that these minicolumns are the entities which the above dipole moment is modeling. The Lagrangian of the SMNI distributions, although possessing multivariate nonlinear means and covariance, have functional forms similar to arguments of firing distributions of individual neurons, so that the description of the columnar dipole above is a model faithful to the standard derivation of a vector potential from an oscillating electric dipole.

Note that this is not necessarily the only or most popular description of electromagnetic influences in neocortex, which 
often describes dendritic presynaptic activity as inducing large scale EEG [21], or axonal firings directly affecting astrocyte processes [22]. This work is only and specifically concerned with electromagnetic fields in collective axonal firings, directly associated with columnar STM phenomena in SMNI calculations, which create vector potentials influencing ion momenta just outside minicolumnar structures.

\section{III. $\mathrm{Ca}^{2+}$}

The roles of $\mathrm{Ca}^{2+}$, while not completely understood, are very well appreciated as being quite important. It is likely that $\mathrm{Ca}^{2+}$ waves are instrumental in tripartite synaptic interactions of astrocytes and neuronal synapses [23]-[25].

\section{A. $\mathrm{Ca}^{2+}$ Momentum}

The momentum at issue is calculated to set the stage for comparison to the vector potential.

In neocortex, a $\mathrm{Ca}^{2+}$ ion with mass $m_{\mathrm{Ca}}=6.6 \times 10^{-26}$ $\mathrm{kg}$, has speed on the order of $50 \mu \mathrm{m} / \mathrm{s}$ [26] to $100 \mu \mathrm{m} / \mathrm{s}$ [25]. This gives a momentum on the order of $10^{-30} \mathrm{~kg}-\mathrm{m} / \mathrm{s}$. An estimate of molar concentrations [25], gives an estimate of a $\mathrm{Ca}^{2+}$ wave as comprised of tens of thousands of such ions.

\section{Vector Potential of EEG Dipoles}

The effective momentum, $\boldsymbol{\Pi}$, affecting the momentum $\mathbf{p}$ of a moving particle in an electromagnetic field, is understood from the canonical momentum [19], [27], [28], in SI units,

$$
\mathbf{\Pi}=\mathbf{p}+q \mathbf{A}
$$

where $q=2 e$ for $\mathrm{Ca}^{2+}, e$ is the magnitude of the charge of an electron $=1.610^{-19} \mathrm{C}$ (Coulomb), and $\mathbf{A}$ is the electromagnetic vector potential. (Note that in Gaussian units $\boldsymbol{\Pi}=\mathbf{p}+q \mathbf{A} / c$, where $c$ is the speed of light.)

$\Pi$ can be used in quantum as well as in classical calculations. Eventually, quantum mechanical calculations including these effects will be performed, as it is clear that in time scales much shorter than neuronal firings $\mathrm{Ca}^{2+}$ wave packets spread over distances the size of typical synapses [29]. Note that gauge of $\mathbf{A}$ is not specified here, and this can lead to important effects especially at quantum scales [18].

For a wire/neuron carrying a current $\mathbf{I}$, measured in $\mathrm{A}=$ Amperes $=\mathrm{C} / \mathrm{s}$,

$$
\mathbf{A}=\frac{\mu}{4 \pi} \int\left(\frac{d r}{r} \mathbf{I}\right)
$$

where the current is along a length $z$ (a neuron), observed from a perpendicular distance $s$. Neglecting far-field retardation effects, this yields

$$
\mathbf{A}=\frac{\mu}{4 \pi} \mathbf{I} \log \left(\frac{z+\left(z^{2}+s^{2}\right)^{1 / 2}}{s}\right)
$$

Other formulae for other geometries are in texts [17]. The point here is the insensitive log dependence on distance. The estimates below assume this log factor to be of order 1 . The magnetic field $\mathbf{B}$ derived from $\mathbf{A}$,

$$
\mathbf{B}=\nabla \times \mathbf{A}
$$

is still attenuated in the glial areas where $\mathrm{Ca}^{2+}$ waves exist, and its magnitude decreases as inverse distance, but $\mathbf{A}$ derived near the minicolumns will be used there and at further distance since it is not so attenuated. The electrical dipole for collective minicolumnar EEG derived from $\mathbf{A}$ is

$$
\mathbf{E}=\frac{i c}{\omega} \nabla \times \mathbf{B}=\frac{i c}{\omega} \nabla \times \nabla \times \mathbf{A}
$$

$\mu_{0}$, the magnetic permeability in vacuum $=4 \pi 10^{-7} \mathrm{H} / \mathrm{m}$ (Henry/meter), where Henry has units of $\mathrm{kg}-\mathrm{m}-\mathrm{C}^{-2}$, is the conversion factor from electrical to mechanical variables. Near neurons, $\mu=10 \mu_{0}$ [30], giving $\mu=10^{-6}$.

$q \mathbf{A}$ can be calculated at several scales:

In studies of small ensembles of neurons [31], an electric dipole moment $\mathbf{Q}$ is defined as $\mathbf{I} z \hat{r}$, where $\hat{r}$ is the direction unit-vector, leading to estimates of $|\mathbf{Q}|$ for a pyramidal neuron on the order of $1 \mathrm{pA}-\mathrm{m}=10^{-12} \mathrm{~A}-\mathrm{m}$. Multiplying by $10^{4}$ synchronous firings in a macrocolumn gives an effective dipole moment $|\mathbf{Q}|=10^{-8}$ A-m. Taking $z$ to be $10^{2} \mu \mathrm{m}=10^{-4} \mathrm{~m}$ (a couple of neocortical layers) to get $\mathbf{I}$, this gives an estimate $|q \mathbf{A}| \approx 2 \times 10^{-19} \times 10^{-6} \times 10^{-8} / 10^{-4}=10^{-27} \mathrm{~kg}-\mathrm{m} / \mathrm{s}$,

Estimates at larger scales [32] give a dipole density $\mathbf{P}=$ $0.1 \mu \mathrm{A} / \mathrm{mm}^{2}$. Multiplying this density by a volume of $\mathrm{mm}^{2} \times$ $10^{2} \mu \mathrm{m}$ (using the same estimate above for $z$ ), gives a $|\mathbf{Q}|=$ $10^{-9} \mathrm{~A}-\mathrm{m}$. This is smaller than that above, due to this estimate including cancellations giving rise to scalp EEG, while the estimate above is within a macrocolumn (the focus of this study), leading to $|q \mathbf{A}|=10^{-28} \mathrm{~kg}-\mathrm{m} / \mathrm{s}$.

\section{SMNI CALCUlations}

\section{A. $\mathrm{Ca}^{2+}$ Momenta}

The time dependence of $\mathrm{Ca}^{2+}$ wave momenta is typically calculated with simulations using code such as NEURON [33], within multivariate differential equations describing interactions among quite a few neuronal elements and parameters. In this study, the resulting flow of $\mathrm{Ca}^{2+}$ wave momenta will be further determined by its interactions in $\Pi$, the canonical momenta which includes $\mathbf{A}$.

\section{B. SMNI-VP}

The outline of coupling the SMNI-VP with $\mathrm{Ca}^{2+}$ waves follows.

Similar to the scaling of mesoscopic columnar firings to an electric potential $\Phi$ describe regional EEG that was fitted to large data sets [15], here columnar firings are scaled to describe the effective current $\mathbf{I}$ giving rise to the vector potential A,

$$
\mathbf{A}=a M^{E} \hat{\mathbf{r}}+b M^{E} \hat{\mathbf{r}}
$$


where $a$ and $b$ are scaled to something on the order of $10^{4} \mathrm{pA}$, as discussed above. $M^{E}$ is the excitatory columnar firing of pyramidal neurons, and $M^{I}$ is the inhibitory columnar firing of pyramidal neurons.

The influence of time-dependent $\mathrm{Ca}^{2+}$ waves is introduced in the post-synaptic and pre-synaptic SMNI parameters, which here also are time-dependent as functions of changing $\mathrm{Ca}^{2+}$ ions.

Such parameters are present at neuronal scales and are included in microscopic NEURON ordinary differential equation calculations. However, as in the original development of SMNI, these parameters are developed to mescolumnar scales.

For example, SMNI mesoscopic firings are described by coupled stochastic differential equations, nonlinear in the drifts and covariance in terms of $M^{E}$ and $M^{I}$ variables, and mesoscopic synaptic and neuronal parameters. It has been most productive to cast these coupled equations into mathematically equivalent conditional probability distributions, which are better suited to handle algebraic intricacies of their rather general nonlinear time-dependent structure, and which afford the use of powerful derivations based on the associated variational principle, e.g., Canonical Momenta Indicators and Euler-Lagrange equations. This is all rigorously discussed and calculated in many preceding SMNI papers. This also required developing powerful numerical algorithms to fit these algebraic models to data [34], [35] and to develop numerical details of the propagating probability distributions using PATHINT [36] and PATHTREE [37].

\section{Coupled SMNI-VP $\mathrm{Ca}^{2+}$ System}

A calculation based on SMNI would incorporate scaled multivariate neuronal processes, developed by NEURON code, into probabilistic descriptions which have numerical codes developed by the author, such as PATHINT and PATHTREE. The Lagrangian, the argument of the exponential defining this probability distribution, includes the canonical energy $\boldsymbol{\Pi}^{2} /\left(2 m_{\mathrm{Ca}}\right)$, which explicitly contains a $\mathbf{p} \cdot \mathbf{A}$ interaction term.

Another alternative, forsaking some power of the probabilistic framework, is to use reasonably accurate most-probable path ordinary differential equation [38], replacing the SMNI stochastic differential equations, to include together with the NEURON calculations.

\section{CONCLUSION}

For several decades the stated Holy Grail of chemical, biological and biophysical research into neocortical information processing has been to reduce such neocortical phenomena into specific bottom-up molecular and smaller-scale processes [39]. Over the past three decades, with regard to short-term memory (STM) and long-term memory (LTM) phenomena, which themselves are likely components of other phenomena like attention and consciousness, the SMNI approach has yielded specific details of STM capacity, duration and stability not present in molecular approaches, but it is clear that most molecular approaches consider it inevitable that their approaches at molecular and possibly even quantum scales will yet prove to be causal explanations of such phenomena.
The SMNI approach is a bottom-up aggregation from synaptic scales to columnar and regional scales of neocortex, and has been merged with larger non-invasive EEG scales with other colleagues - all at scales much coarser than molecular scales. As with many Crusades for some truths, other truths can be trampled. It is proposed that an SMNI vector potential (SMNIVP) constructed from magnetic fields induced by neuronal electrical firings, at thresholds of collective minicolumnar activity with laminar specification, can give rise to causal top-down mechanisms that effect molecular excitatory and inhibitory processes in STM and LTM. Such a smoking gun for top-down effects awaits forensic in vivo experimental verification, requiring appreciating the necessity and due diligence of including true multiple-scale interactions across orders of magnitude in the complex neocortical environment.

While many studies have examined the influences of changes in $\mathrm{Ca}^{2+}$ distributions on large-scale EEG [40], there have not been studies examining the complimentary effects on $\mathrm{Ca}^{2+}$ ions at a given neuron site from EEG-induced magnetic fields arising from other neuron sites.

Thus, a single $\mathrm{Ca}^{2+}$ ion can have a momentum appreciably altered in the presence of macrocolumnar EEG firings, and this effect is magnified when many ions in a wave are similarly affected. Therefore, large-scale top-down neocortical processing giving rise to measurable scalp EEG can directly influence atomic-scale bottom-up processes.

This suggests that, instead of the common assumption that $\mathrm{Ca}^{2+}$ waves contribute to neuronal activity, they may in fact at times be caused by the influence of $\mathbf{A}$ of larger-scale EEG. Such a "smoking gun" for top-down effects awaits forensic in vivo experimental verification, requiring appreciating the necessity and due diligence of including true multiple-scale interactions across orders of magnitude in the complex neocortical environment.

\section{ACKNOWLEDGMENT}

I thank Paul Nunez and William Ross for verification of some experimental data.

\section{REFERENCES}

[1] C. Anastassiou, R. Perin, H. Markram, and C. Koch, "Ephaptic coupling of cortical neurons," Nature Neuroscience, vol. 14, pp. 217-223, 2011.

[2] P. Nunez, R. Srinivasan, and L. Ingber, "Theoretical and experimental electrophysiology in human neocortex: Multiscale correlates of conscious experience," in Multiscale Analysis and Nonlinear Dynamics, M. Pesenson, Ed. New York: Wiley, 2012, p. (to be published).

[3] R. Silberstein, "Neuromodulation of neocortical dynamics," in Neocortical Dynamics and Human EEG Rhythms, P. Nunez, Ed. New York, NY: Oxford University Press, 1995, pp. 628-681.

[4] I. Kominis, "Zeno is pro Darwin: quantum Zeno effect suppresses the dependence of radical-ion-pair reaction yields on exchange and dipolar interactions," University of Crete, Greece, Tech. Rep. arXiv:0908.0763v2 [quant-ph], 2009.

[5] C. Rodgers and P. Hore, "Chemical magnetoreception in birds: The radical pair mechanism," PNAS, vol. 106, no. 2, pp. 353-360, 2009.

[6] I. Solov'yov and K. Schulten, "Magnetoreception through cryptochrome may involve superoxide," Biophys. J., vol. 96, no. 12, pp. 4804-4813, 2009.

[7] S. Johnsen and K. Lohmann, "Magnetoreception in animals," Phys. Today, vol. 61, pp. 29-35, 2008.

[8] G. Gordon, K. Iremonger, S. Kantevari, G. Ellis Davies, B. MacVicar, and J. Bains, "Astrocyte-mediated distributed plasticity at hypothalamic glutamate synapses," Neuron, vol. 64, pp. 391-403, 2009. 
[9] M. Banaclocha, "Neuromagnetic dialogue between neuronal minicolumns and astroglial network: A new approach for memory and cerebral computation," Brain Res. Bull., vol. 73, pp. 21-27, 2007.

[10] J. A. Pereira and F. Furlan, "Astrocytes and human cognition: Modeling information integration and modulation of neuronal activity," Progress in Neurobiology, vol. 92, pp. 405-420, 2010.

[11] L. Ingber, "Statistical mechanics of neocortical interactions. I. Basic formulation," Physica D, vol. 5, pp. 83-107, 1982, http://www.ingber. com/smni82\_basic.pdf

[12] L. Ingber, "Statistical mechanics of neocortical interactions. Dynamics of synaptic modification," Phys. Rev. A, vol. 28, pp. 395-416, 1983, http://www.ingber.com/smni83 \_dynamics.pdf.

[13] L. Ingber, "Statistical mechanics of neocortical interactions. Derivation of short-term-memory capacity," Phys. Rev. A, vol. 29, pp. 3346-3358, 1984, http://www.ingber.com/smni84\_stm.pdf.

[14] L. Ingber, "Statistical mechanics of neocortical interactions: Pathintegral evolution of short-term memory," Phys. Rev. E, vol. 49, no. 5B, pp. 4652-4664, 1994, http://www.ingber.com/smni94\_stm.pdf.

[15] L. Ingber, "Statistical mechanics of neocortical interactions: Applications of canonical momenta indicators to electroencephalography," Phys. Rev. E, vol. 55, no. 4, pp. 4578-4593, 1997, http://www.ingber.com/ smni97\_cmi.pdf.

[16] L. Ingber, "Columnar EEG magnetic influences on molecular development of short-term memory," in Short-Term Memory: New Research, G. Kalivas and S. Petralia, Eds. Hauppauge, NY: Nova, 2012a, pp. 37-72, Invited Paper. http://www.ingber.com/smni11 \_stm \_scales.pdf.

[17] J. Jackson, Classical Electrodynamics. New York: Wiley \& Sons, 1962.

[18] J. Tollaksen, Y. Aharonov, A. Casher, T. Kaufherr, and S. Nussinov, "Quantum interference experiments, modular variables and weak measurements," New J. Phys., vol. 12, no. 013023, pp. 1-29, 2010.

[19] R. Feynman, R. Leighton, and M. Sands, The Feynman Lectures on Physics. Reading, MA: Addison-Wesley, 1964.

[20] G. Giuliani, "Vector potential, electromagnetic induction and 'physical meaning'," Eur. J. Phys., vol. 31, no. 4, pp. 871-880, 2010.

[21] P. Nunez, Electric Fields of the Brain: The Neurophysics of EEG. London: Oxford University Press, 1981.

[22] J. McFadden, "Conscious electromagnetic field theory," NeuroQuantology, vol. 5, no. 3, pp. 262-270, 2007.

[23] C. Agulhon, J. Petravicz, A. McMullen, E. Sweger, S. Minton, S. Taves, K. Casper, T. Fiacco, and K. McCarthy, "What is the role of astrocyte calcium in neurophysiology?" Neuron, vol. 59, pp. 932-946, 2008.

[24] A. Araque and M. Navarrete, "Glial cells in neuronal network function," Phil. Tran. R. Soc. B, pp. 2375-2381, 2010.

[25] W. Ross, "Understanding calcium waves and sparks in central neurons," Nature, vol. 13, pp. 157-168, 2012.

[26] S. Bellinger, "Modeling calcium wave oscillations in astrocytes," $\mathrm{Neu}$ rocomputing, vol. 65, no. 66, pp. 843-850, 2005.

[27] R. Feynman, Quantum Electrodynamics. New York: W.A. Benjamin, 1961.

[28] H. Goldstein, Classical Mechanics, 2nd ed. Reading, MA: Addison Wesley, 1980

[29] H. Stapp, Mind, Matter and Quantum Mechanics. New York: SpringerVerlag, 1993.

[30] D. Georgiev, "Electric and magnetic fields inside neurons and their impact upon the cytoskeletal microtubules," Cogprints, U. Southampton, UK, Tech. Rep. Cogprints Report, 2003, http://cogprints.org/3190/.

[31] S. Murakami and Y. Okada, "Contributions of principal neocortical neurons to magnetoencephalography and electroencephalography signals," J. Physiol., vol. 575, no. 3, pp. 925-936, 2006.

[32] P. Nunez and R. Srinivasan, Electric Fields of the Brain: The Neurophysics of EEG, 2nd Ed. London: Oxford University Press, 2006.

[33] N. Carnevale and M. Hines, The NEURON Book. Cambridge, UK: Cambridge U Press, 2006.

[34] L. Ingber, "Adaptive Simulated Annealing (ASA)," Caltech Alumni Association, Pasadena, CA, Tech. Rep. Global optimization C-code, 1993, http://www.ingber.com/ \\#ASA-CODE.

[35] L. Ingber, "Adaptive Simulated Annealing," in Stochastic global optimization and its applications with fuzzy adaptive simulated annealing, J. H.A. Oliveira, A. Petraglia, L. Ingber, M. Machado, and M. Petraglia, Eds. New York: Springer, 2012b, pp. 33-61, Invited Paper. http://www.ingber.com/asa11 \_options.pdf.

[36] L. Ingber and P. Nunez, "Statistical mechanics of neocortical interactions: High resolution path-integral calculation of short-term memory," Phys. Rev. E, vol. 51, no. 5, pp. 5074-5083, 1995, http://www.ingber. com/smni95\_stm.pdf.
[37] L. Ingber, C. Chen, R. Mondescu, D. Muzzall, and M. Renedo, "Probability tree algorithm for general diffusion processes," Phys. Rev. $E$, vol. 64, no. 5, pp. 056 702-056 707, 2001, http://www.ingber.com/ path01 \_pathtree.pdf.

[38] H. Dekker, "On the most probable transition path of a general diffusion process," Phys. Lett. A, vol. 80, pp. 99-101, 1980.

[39] M. Rabinovich, P. Varona, A. Selverston, and H. Arbaranel, "Dynamical principles in neuroscience," Rev. Mod. Phys., vol. 78, no. 4, pp. 12131265, 2006.

[40] P. Kudela, G. Bergey, and P. Franaszczuk, "Calcium involvement in regulation of neuronal bursting in disinhibited neuronal networks: Insights from calcium studies in a spherical cell model," Biophys. J., vol. 97, no. 12 , pp. 3065-3074, 2009.

Lester Ingber received: his diploma from Brooklyn Technical High School in 1958; his B.S. in physics from Caltech in 1962; his Ph.D. in theoretical nuclear physics from UC San Diego in 1966, while studying at the Niels Bohr Institute in Copenhagen and consulting at RAND in Santa Monica CA.

He has published over 100 papers and books in theoretical nuclear physics, neuroscience, finance, general optimization, combat analysis, karate, and education. Through Lester Ingber Research (LIR) in Ashland OR he develops and consults on projects documented in the http://www.ingber.com/ archive.

Prof. Ingber has held positions in academia, government and industry: National Science Foundation Postdoctoral Fellow at UC Berkeley and UC Los Angeles; Assistant Professor in physics at SUNY at Stony Brook; Research Physicist in the Physics department and in the Institute for Pure and Applied Physical Sciences at UC San Diego; Research Associate at UC San Diego in the Music department; twice Senior Research Associate of the National Research Council of the National Academy of Sciences; Professor of Physics at the Naval Postgraduate School in Monterey CA and the US Army Concepts Analysis Agency in Bethesda MD; Research Professor of Mathematics at The George Washington University in DC; Director of Research and Development at trading firm DRW in Chicago IL and hedge fund DUNN Capital Management in Stuart FL; Partner in Pion Capital, a hedge-fund partnership of Caltech alumni; Editor-in-Chief at Research Publisher for: Current Progress Journal (timely topics in science), Graduate Journal of Research, and Undergraduate Journal of Research, and associated e-conferences for these three journals. 